IOSR Journal of Pharmacy

ISSN: 2250-3013, www.iosrphr.org

|| Volume 2 Issue 5 || Sep-Oct. 2012 || PP.51-55

\title{
Prevalence of microalbuminuria among sudanese type 2 diabetic patients at elmusbah center at ombadda - omdurman
}

\author{
Fayza Ahmed Rahamtalla', Atif A. Elagib ${ }^{2}$, Ahmed Mahdi ${ }^{3}$, \\ And Samia Mahdi Ahmed ${ }^{4}$ \\ ${ }^{1}$ Lecturer, Department of Clinical Chemistry, Faculty of Medical Laboratory Sciences Omdurman \\ Islamic University, Khartoum, Sudan \\ ${ }^{2}$ Associate professor, Department of Immunology and Biotechnology; Tropical Medicine research \\ Institute (NCR), Khartoum, Sudan \\ ${ }^{3}$ Assisstant professor, Department of Clinical Chemistry, Faculty of Medical Laboratory Sciences \\ Omdurman Islamic University, Khartoum, Sudan \\ ${ }^{4}$ Assistant professor, Department of Clinical Chemistry, Faculty of Medical Laboratory Sciences \\ Sudan University of Science and Technology, Khartoum, Sudan
}

\begin{abstract}
Microalbuminuria is persistent albumin excretion between 30 and $300 \mathrm{mg} /$ day, in patients with diabetes it is usually indicative of diabetic nephropathy which is most common cause of end stage renal disease, it is also associated with cardiovascular disease. Early detection of microalbuminuria and early control of diabetes retards the development of structural changes in early diabetic nephropathy.

The main objective was to determine the prevalence of microalbuminuria on urine samples of Sudanese type 2 diabetic patients (non- insulin-dependent) which is expressed by Albumin creatinine ratio (ACR).

Across- sectional hospital based study was carried out in Elmusbah Medical Center, from November 2008 to March 2009, fifty eight of type 2 diabetic patients studied included 29 females (aged 35 - 80 years) and 29 males (aged 43 - 88 years). Urinary albumin concentration was measured by immunoturbodimetric assay. Microalbuminuria was diagnosed in 26 (44\%) patients. The prevalence of microalbuminuria was $8.66 \%$ from total populations at risk ( $\mathrm{N}=300)$.

The risk factors associated with microalbuminuria were found to be age, duration of diabetes, systolic and diastolic blood pressure.
\end{abstract}

Keywords-Albumin creatinine ratio (ACR), Microalbuminuria, Type 2diabetes mellitus

\section{INTRODUCTION}

The prevalence of type 2 diabetes mellitus has been increasing significantly in all countries during the last century. By 2010, 220 million people in the world are projected to be afflicted by this disease (1).

The importance of protecting the body from hyperglycemia cannot be overstated. The injurious effects of hyperglycemia are diabetic nephropathy, neuropathy, retinopathy and cardiovascular disease (2)

One of the earliest markers of diabetic nephropathy is the presence of microalbuminuria (MAU), this term was identify and differentiate albumin excretion rates ( 20 to $200 \mu \mathrm{g} / \mathrm{min}$ ) that were below usual level of detection by a standard dipstick method (>200 $\mu \mathrm{g} / \mathrm{min}$, also termed macroalbuminuria) in patients with diabetes. The presence of macroalbuminuria indicates more serious kidney disease, progression to end stage renal disease (ESRD) which may then occurs within several years (3.4).

Once overt kidney failure has developed two years survival is approximately $50 \%$, MAU is associated also with cardiovascular disease in patients with diabetes and hypertension. Type 2 diabetics with MAU have a $50 \%$ chance of having a coronary event within 7 years. In type 2, having microalbuminuria is a powerful message that they have an increasing risk of heart disease, which reflects vascular damage and appears to be a marker of early arterial disease and endothelial dysfunction (5.6). The American Diabetes Association (ADA) recommended that people with diabetes should do an annual microalbuminuria urine test and measurement blood creatinine at least once a year. The ratio of albumin to creatinine is usually less than $2.0 \mathrm{mg} / \mathrm{mmol}$ for men (and $2.8 \mathrm{mg} / \mathrm{mmol}$ for women 7 ). 


\section{MATERIALS AND METHODS}

Across sectional hospital based study was carried out in Elmusbah Medical Center- Ombadda, Omdurman province, Khartoum state, from November 2008 to March 2009. Volunteers participated in this study after taking informed consents. Fifty-eight type 2 (NIDD) patients were enrolled in this study; 29 were females (aged 35 - 80 years), while 29 were males (aged 43 - 88 years). Also, fifty- eight healthy non-diabetic nonhypertensive healthy controls, 29 were males (aged 39 - 72 years), while 29 were females (aged 33 - 67 years), from Omdurman province. Patients with urinary tract infection (UTI), heart failure, and patients with positive urine dipstick for protein were excluded from the study.

In all patients, a clinical check were done, including height, weight, and the body mass index was calculated, the blood pressure was recorded.

Early morning urine samples from all patients and healthy control were collected in sterile urine containers. The urine samples were centrifuged to get supernatant (creatinine urine samples were diluted with distilled water 1/50). Then microalbuminuria levels were measured using A15 Biosystems analyzer, the method used was Immunoturbidometric assay (8.9). Creatinine levels were measured by using A15 BioSystem analyzer, the method used was Jaffe method (10.11).

Fasting blood sample was drawn after ten hours overnight fast, and collected in vials containing fluoride/oxalate, then glucose levels were measured using CHEM 7 from ERBA, the method used was enzymatic colorimetric end point method (12.13).

Data were statistically analyzed using SPSS Computer program. The analysis of variance and the differences among the means were done for significance at 5\% level using Mann-Whitney test, the prevalence were calculated using this formula: prevalence $=$ all patients have disease/total population at risk at a period of time, the relationship between variables were determined using correlation analysis, and Linear regression analysis also used to assess association between albumin/creatinine ratio (ACR) as dependent variables and Systolic blood pressure (SBP) as independent variable.

\section{III.RESULTS}

3.1. Prevalence of microalbuminuria in Sudanese diabetic patients

The prevalence of microalbuminuria among Sudanese diabetic patient was $8.66 \%$ from total population at risk (300). Overall microalbuminuria was detected in $44 \%$ of the diabetic patient. The ACR among males represented $51.7 \%$ while among females was $37.9 \%$.

\subsection{Albumin/Creatinine ratio (ACR)}

The mean ACR in male patients was $5.97 \pm 7.68(\mathrm{mg} / \mathrm{mmol})$, while it was $10.54 \pm 37.77(\mathrm{mg} / \mathrm{mmol})$ in females patients. The mean ACR was significantly higher in Sudanese diabetic males \& females compared with healthy control groups $(\mathrm{P}=0.000 \& \mathrm{P}=0.004$ respectively), by applying Mann-Whitney test (Table1).

Table 1: The mean and standard deviation of ACR among diabetic patients and healthy controls

\begin{tabular}{|r|r|r|r|r|r|r|}
\hline $\begin{array}{l}\text { ACR } \\
(\mathrm{mg} / \mathrm{mmol})\end{array}$ & $\begin{array}{r}\text { Male } \\
\text { patients }\end{array}$ & $\begin{array}{r}\text { Male } \\
\text { healthy } \\
\text { control }\end{array}$ & P Value & $\begin{array}{r}\text { Female } \\
\text { patients }\end{array}$ & $\begin{array}{r}\text { Female } \\
\text { healthy } \\
\text { controls }\end{array}$ & P Value \\
\hline Mean & 5.97 & 0.562 & 0.000 & 10.54 & 0.733 & 0.004 \\
\hline SD & 7.68 & 0.26 & & 37.77 & 1.82 & \\
\hline
\end{tabular}

There was significant difference in the number of patients with high ACR and patients with normal ACR in both males and females $(\mathrm{P}=0.025 \& \mathrm{P}=0.000$ respectively), by Wilcoxon sign ranks test (Table 2 ).

Table 2: The comparison between number of patients with \& without microalbuminuria 


\begin{tabular}{|r|rr|r|r|r|}
\hline Number & $\begin{array}{r}\text { Patients } \\
\text { microalbuminuria }\end{array}$ & $\begin{array}{r}\text { Patients } \begin{array}{r}\text { without } \\
\text { microalbuminuria }\end{array} \\
15\end{array} \quad \begin{array}{r}\text { Total } \\
\text { number }\end{array}$ & P-Value \\
\hline Male & 11 & 13 & 28 & 0.025 \\
\hline Females & 11 & 29 & 0.000 \\
\hline
\end{tabular}

* One missing male

\subsection{Risk factors associated with high microalbuminuria in Sudanese diabetic patients}

In males patients age and age of onset were positively correlated $(\mathrm{P}=0.000)$, age and duration of diabetes were positively correlated $(\mathrm{P}=0.006)$ also smoking and complications of diabetes was positively correlated $(P=0.000)$ but smoking and body mass index $(\mathrm{BMI})$ was negatively correlated $(\mathrm{P}=0.007)$, $\mathrm{BMI}$ and complications is positively correlated $(\mathrm{P}=0.011)$, also ACR and Systolic blood pressure (SBP) was positively correlated $(\mathrm{P}=0.030)$ (Table 3).

Table 3: Risk factors associated with diabetes among males

\begin{tabular}{|r|r|r|}
\hline \multicolumn{1}{|l|}{ Factors } & Factors & Sig(2-tailed) \\
\hline Age & Age of onset & 0.000 \\
\hline Age & Duration & 0.006 \\
\hline Smoking & Complication & 0.000 \\
\hline Smoking & BMI & 0.007 \\
\hline BMI & Complication & 0.011 \\
\hline SBP & DBP & 0.000 \\
\hline ACR & SBP & 0.030 \\
\hline
\end{tabular}

In female patients age and age of onset was positively correlated $(\mathrm{P}=0.000)$, age was positively correlated with duration of diabetes $(\mathrm{P}=0.001)$ and with ACR ( $\mathrm{P}=0.025)$, duration and fasting blood sugar (FBS) was positively correlated ( $\mathrm{P}=0.014)$, also duration is positively correlated with ACR $(\mathrm{P}=0.013$ ), systolic blood pressure $(\mathrm{SBP})$ and diastolic blood pressure $(\mathrm{DBP})$ was positively correlated $(\mathrm{P}=0.002)$, also SBP is positively correlated with ACR ( $\mathrm{P}=0.034)$ (Table 4).

Table 4: Risk factors associated with diabetes among females 


\begin{tabular}{|r|r|r|}
\hline Factors & Factors & Sig(2-tailed) \\
\hline Age & Age of onset & 0.000 \\
\hline Age & Duration & 0.001 \\
\hline FBS & Duration & 0.014 \\
\hline SBP & DBP & 0.002 \\
\hline ACR & SBP & 0.034 \\
\hline ACR & Age & 0.025 \\
\hline ACR & Duration & 0.013 \\
\hline
\end{tabular}

The correlation between the systolic blood pressure (SBP) and Albumin/Creatinine ratio (ACR), was performed by the regression analysis:

$\mathrm{ACR}=-25.703+.255 * \mathrm{SBP}(\mathrm{P}=0.03)$. So ACR could be best explained by SBP.

\section{DISCUSSION}

In the present study, the prevalence of diabetic microalbuminuria in 58 type 2 diabetic patients was found to be $8.66 \%$. This was Similar to the worldwide prevalence which varies from 5 to $20 \%$ (14). Studies in the white UK population revealed a prevalence of microalbuminuria of 7-9\%, while in Mexican American, it was $30 \%$, this variation in prevalence can be attributed to factors such as variation in albumin excretion (i.e between 30 and $300 \mathrm{mg} / \mathrm{day}$ ), method of urine collection or difference in ethnic susceptibility.

Microalbuminuria is useful predictor of renal failure in patients with diabetes, and even an independent predictor of mortality in type 2 diabetes (15).

The causal risks factors for microalbuminuria are raised blood pressure and poor glycaemic control. Some studies have revealed duration of the diabetes, male sex, and retinopathy as major risk factors for microalbuminuria (16).

Increased blood pressure, particularly systolic blood pressure, induces systemic artiole dysfunction in the kidney and damage to glomerrular filtration membrane; this is most important reason for increased urine protein excretory rate (17).

Analysis of this study revealed that, the age, duration of diabetes, systolic and diastolic blood pressure as the risk factors for microalbuminuria. The majority of patients were hypertensive (60\% males and $45 \%$ females). Microalbuminuria was found to be more common among males compared with females, which is similar to other results of McLaughlin (18).

Regarding fasting blood sugar the findings were positively correlated in female populations of the study (i.e. there were significant difference between patients with and without microalbuminuria), but however in the males group the finding was far different, as no differences were elucidated among patients with and without microalbuminuria, and this may be attributed to a wide variety of factors. In spite of this, FBS remains a crucial and determinant factor in this issue of concern in microalbuminuria.

Microalbuminuria is now recognized as an independent cardiac risk factor even in the absence of diabetes (19), so that microalbuminuria can be used as a marker for endothelial dysfunction, vascular injury, renal disease, and cardiovascular disease (20).

Other factor which reported to be associated with microalbuminuria was the smoking (21), analysis of this study revealed that no association was found between smoking and microalbuminuria, which agreed with Chase (22), this variation in finding can be attributed to factors such as number of smokers, lifestyle, and genetic make up.

Considering treatment, only $6.7 \%$ of males and $9.1 \%$ of females were treated with insulin and the majority with oral hypoglycemic drugs. In patients with detected microalbuminuria, sulfonylurea and insulin secretagogues are usually not very effective due to low endogenous production of insulin resulting from the long duration of diabetes. Thus, most type 2 diabetic patients with diabetes nephropathy should be treated with insulin as reported by Jorge [23].

\section{V.CONCLUSION}

The prevalence of microalbuminuria in our study was found to be $8.66 \%$ and the risk factors are similar to that reported in other studies. These findings encourage us for tight blood glucose control to reduce the burden of diabetic kidney disease in the future. 


\section{REFERENCES}

[1]. A.F. Amos, D.J. McCarthy, P. Zimmet. The rising global burden of diabetes and its complications: estimates and projections to the year 2010. Diabet Med 1997; 14 (Suppl 5): S1-S85.

[2]. J. Michael, M.D. Fowler. Microvascular and Macrovascular Complications of Diabetes, Diabetes Foundation, Clinical Diabetes 2008; 26 (2).

[3]. P.A. Svendsen, B. Oxenball, J.S. Christinsen. Microalbuminuria in diabetic patients: A longitudinal study. Crinol Suppl (Copenh) 1981; 242: 53-54.

[4]. G.C. Vibertiz, R.D. Hill, R.J. Jarrett, et al. Microalbuminuria as a predictor of clinical nephropathy in insulin dependent diabetes mellitus. Lancet 1982; 1: 1430-1432.

[5]. B.D. Rose, G.L. Bakris. Microalbuminuria and Cardiovascular disease Wellesley, Diabetes Care 2004; 28 (1): 164-76.

[6]. D.M. Nathan, P.A. Cleary, J.Y. 1. Backlund. Intensive diabetes treatment and cardiovascular disease in patients with type 1 diabetes $N$ Engl J Med 2005; 353: 2643-2653.

[7]. American Diabetes Association, Standards of Medical Care in Diabetes, 2008.

[8]. C.L. Cambiaso, D. Collet-Cas Sart, M. Lievens. Immunoassay of low concentration of albumin in urine by latex particle counting. Clin Chem 1988; 34(2): 416-418.

[9]. E.A. Medcalf, D.J. Newman, E.G. Gorman, et al. Rapid, robust method for measuring low concentrations of albumin in urine. Clin Chem 1990; 36(3): 446-449.

[10]. D.L. Fabiny, G. Ertingshausen. Automated reaction-rate method for determination of serum creatinine with centrifichem. Clin. Chem 1971; 17: 696-700.

[11]. C.A. Bursts, E.R. Ashwood, D.E. Bruns. Tietz Textbook of clinical chemistry, $3^{\text {rd }}$ edition. Saunders Co., 1999.

[12]. D. Barham, P. Trinder. Analyst 1972; 97: 142.

[13]. B. Szasz, k. Hurt, E. Busch. Tropic Cli. Chem. Cli. Biochem1974; 12: 256.

[14]. A.L Adler, R.J Stevens, S.E Manley. Development and progression of nephropathy in type2 diabetes: the United Kingdom Prospective Diabetes Study 2003; 63: 225-232.

[15]. A. Neil, M. Hawkins, M. Potok, et al. Prospective population - based study of microalbuminuria as a predictor of mortality in NIDDM. Diabetes Care 1993; 16: 996-1003.

[16]. R. Klein. Prevalence of microalbuminuria in older onset diabetes. Diabetes Care 1993; 16: 1325-29.

[17]. R. Pedrinelli, G. Dell'Omo, G. Penno, et al. Microalbuminuria and pulse pressure in hypertensive and atherosclerotic men. Hypertension 2000; 35: 48-54.

[18]. K. Mclaughlin, Albuminuria, a Review article. E-Medicine Specialties, 2001.

[19]. UK Prospective Diabetes Study Group. Intensive blood-glucose control with sulphonylureas or insulin compared with conventional treatment and risk of complications in patients with type2 diabetes. Lancet 1998; 352: 837-853.

[20]. P. Nelson, D. O. Kopyt. Slowing Progression along the Renal Disease continuum. JAOA 2005; 105: 207-215.

[21]. M.A. Gall, Prevalence of micro- and microalbuminuria, arterial hypertension, retinopathy, and large vessels disease in European type 2 diabetic patients. Diabetologia 1991; 34: 655-61.

[22]. H.P. Chase Cigarette smoking increases the risk of albuminuria among subjects with type1 diabetes. JAMA 1991; 265: 614-617.

[23]. L.G Jorge, J. Mirela de Azevedo, P.S silveiro. Diabetic Nephropathy: Diagnosis, Prevention, and Treatment Care 2005; 28: 164-176. 